\title{
Hypokalemia in Patients with COVID-19
}

\author{
Gaetano Alfano ${ }^{1,2,3} \cdot$ Annachiara Ferrari $^{1}$ - Francesco Fontana ${ }^{2} \cdot$ Rossella Perrone $^{1}$. Giacomo Mori ${ }^{2}$. \\ Elisabetta Ascione ${ }^{2}$. Riccardo Magistroni ${ }^{1,2}$. Giulia Venturi ${ }^{4}$. Simone Pederzoli ${ }^{5}$. Gianluca Margiotta ${ }^{5}$. \\ Marilina Romeo ${ }^{5} \cdot$ Francesca Piccinini $^{5} \cdot$ Giacomo Franceschi $^{6} \cdot$ Sara Volpi $^{6} \cdot$ Matteo Faltoni $^{6} \cdot$ Giacomo Ciusa $^{6}$. \\ Erica Bacca ${ }^{6} \cdot$ Marco Tutone $^{6}$. Alessandro Raimondi ${ }^{6}$. Marianna Menozzi ${ }^{6}$. Erica Franceschini ${ }^{6} \cdot$ Gianluca Cuomo $^{6}$. \\ Gabriella Orlando ${ }^{6}$. Antonella Santoro ${ }^{6}$. Margherita Di Gaetano ${ }^{6}$. Cinzia Puzzolante ${ }^{6}$. Federica Carli ${ }^{6}$. \\ Andrea Bedini ${ }^{6} \cdot$ Jovana Milic $^{3,6} \cdot$ Marianna Meschiari ${ }^{6} \cdot$ Cristina Mussini $^{6} \cdot$ Gianni Cappelli $^{1,2} \cdot$ Giovanni Guaraldi $^{6}$ on \\ behalf of the Modena Covid-19 Working Group (MoCo19)
}

Received: 24 August 2020 / Accepted: 12 November 2020 / Published online: 4 January 2021

(c) Japanese Society of Nephrology 2021

\begin{abstract}
Background Patients with COVID-19 experience multiple clinical conditions that may cause electrolyte imbalances. Hypokalemia is a concerning electrolyte disorder closely associated with severe complications. This study aimed to estimate prevalence, risk factors and outcome of hypokalemia in a cohort of patients with confirmed COVID-19.

Methods A retrospective analysis was conducted on 290 non-ICU admitted patients with COVID-19 at the tertiary teaching hospital of Modena, Italy, from February 16 to April 14, 2020.

Results Hypokalemia was detected in 119 out of 290 patients (41\%) during hospitalization. Mean serum potassium was $3.1 \pm 0.1 \mathrm{meq} / \mathrm{L}$. The majority of patients $(90.7 \%)$ patients experienced only a mild decrease in serum potassium level (3-3.4 mEq/L). Hypokalemia was associated with hypocalcemia, which was detected in $50 \%$ of subjects. Urine potassiumto-creatinine ratio, measured in a small number of patients $(n=45 ; 36.1 \%)$, revealed an increase of urinary potassium excretion in most cases $(95.5 \%)$.

Risk factors for hypokalemia were female sex (odds ratio (OR) 2.44; 95\% CI 1.36-4.37; $P$ 0.003) and diuretic therapy (OR $1.94,95 \%$ CI 1.08-3.48; $P$ 0.027). Hypokalemia, adjusted for sex, age and SOFA score, was not associated with ICU transfer (OR 0.52; 95\% CI 0.228-1.212; $P=0.131$ ), in-hospital mortality (OR, 0.47; 95\% CI 0.170-1.324; $\mathrm{P}=0.154$ ) and composite outcome of ICU transfer or in-hospital mortality (OR $0.48 ; 95 \%$ CI $0.222-1.047 ; P=0.065$ ) in our cohort of patients.

Conclusions Hypokalemia was a frequent disorder in subjects with COVID-19. Female sex and diuretic therapy were identified as risk factors for low serum potassium levels. Hypokalemia was unrelated to ICU transfer and death in this cohort of patients.
\end{abstract}

Keywords Coronavirus $\cdot$ COVID $\cdot$ Electrolytes $\cdot$ Hypokalemia $\cdot$ Potassium $\cdot$ Magnesium

Modena Covid-19 Working Group (MoCo19) details are given in acknowledgement.

Electronic supplementary material The online version of this article (https://doi.org/10.1007/s10157-020-01996-4) contains supplementary material, which is available to authorized users.

Gaetano Alfano

gaetano.alfano@unimore.it

Extended author information available on the last page of the article

\section{Introduction}

COVID-19 is an emerging pandemic spreading worldwide from December 2019. On through 27 October 2020, the death of more than one million people and the increase in the number of newly infected people are posing a threat to global health [1].

Epidemiological studies documented that the majority of COVID-19 cases has a mild self-limited presentation (81\%) but the development of severe respiratory symptoms (14\%) such as dyspnea, tachypnea and hypoxia require hospital admission [2]. The lung is the principal target of the novel 
coronavirus, and atypical pneumonia is the most common clinical presentation [3]. Multiple organs such as the heart, brain, liver and kidneys can be involved during this infection [4]. Systemic release of cytokines is thought to be the cause of organ dysfunction and, therefore, the major determinant of morbidity in these patients [5]. Cytokines provoke a severe pro-inflammatory state leading to hypoxemia and sepsis requiring intensification of supportive therapy. Generally, hospitalized patients belong to a vulnerable subset of the population because they are older adults and have several underlying medical conditions [6]. Patients with COVID-19 can experience a long hospital stay. They usually undergo multiple treatments varying from delivering of ventilatory support to the administration of experimental agents for SARS-CoV-2 infection. In this setting, fever, hyperventilation, sweating, medication-related side effects and dietary changes may cause concerning electrolyte imbalances.

Maturing experience in the care of COVID-19 patients showed that hypokalemia is a frequent lab abnormality. There is particular concern about this disorder as it may increase the susceptibility to potential fatal arrhythmia in COVID-19 patients [7, 8]. Hypokalemia has been described in a few case-series during the SARS-Cov- 1 outbreak in 2003, but there is a lack of data on the exact mechanisms underlying this disorder $[9,10]$. Possible causes of hypokalemia in the context of SARS-CoV2 infection may result from hyper-activation of the renin-angiotensin-aldosterone system (RAAS), gastrointestinal losses, anorexia secondary to concurrent illness and tubular damage caused by ischemia or nephrotoxicagents. With regard to this latter hypothesis, tubular damage may be linked to the direct cytotoxic effect of SARS-CoV-2, since the virus has been associate to diffuse tubular damage [11].

Considering these unanswered clinical questions, we evaluated a cohort of hospitalized patients to assess the prevalence of hypokalemia and its impact on clinical outcomes.

\section{Methods}

\section{Study design}

A single-center, retrospective, observational study was conducted at the University Hospital of Modena. We retrospectively reviewed all the electronic records of 320 noncritically ill patients admitted to our hospital from February 16 to April 14, 2020, with a diagnosis of SARS-CoV-2 infection, according to WHO interim guidelines [12].

The study was approved by the regional ethical committee of Emilia Romagna (prot. n. 0013376/20).

\section{Data collection}

We collected data on age, sex, laboratory values during admission (hemoglobin concentration, lymphocyte count, platelet count, arterial blood gas analysis, markers of inflammation, serum and urine creatinine, urea and urine electrolyte), comorbidities, sign and symptoms at presentation, vital signs and administered treatment (diuretic, electrolyte supplement). Rates of ICU transfer and living status at the end of the observation period were used as primary outcome variables.

\section{Outcomes measures}

The primary objective of our study was to evaluate the prevalence of hypokalemia. Secondary outcomes included assessment of hypokalemia on the risk of ICU transfer and in-hospital mortality of non-ICU admitted patients.

\section{Variables and definition}

Hypokalemia was defined as a serum potassium level $<3.5 \mathrm{mEq} / \mathrm{l}$. The normal level of serum potassium ranges from 3.5 to $5.3 \mathrm{mEq} / \mathrm{L}$. The diagnosis of hypokalemia was performed on a single value of serum potassium $<3.5 \mathrm{meq} / \mathrm{L}$ at any time during hospitalization. We excluded all serum values of potassium measured on plasma and by blood gas analyzer.

Severity was classified as mild when the serum potassium level was 3-3.4 mmol/L, moderate when the serum potassium level was $2.5-3 \mathrm{mmol} / \mathrm{L}$, and severe when the serum potassium level was less than $2.5 \mathrm{mmol} / \mathrm{L}$.

Hypocalcemia and hypomagnesemia were defined by a serum level of less than $8.5 \mathrm{mg} / \mathrm{dl}$ and $1.6 \mathrm{mg} / \mathrm{dl}$, respectively. Acute kidney injury (AKI) was defined according to the 2012 Kidney Disease: Improving Global Outcomes (KDIGO) guidelines [13].

Potassium urinary excretion was measured as urine potassium-to-creatinine ratio. A value of potassium urinary excretion $>1.5$ indicated inappropriate renal potassium loss [14-16]. Potassium urinary excretion was not measured in patients with chronic and acute kidney disease.

Sodium urinary excretion was measured with the fractional excretion of sodium ( $\mathrm{FeNa}$ ); a value $<1 \%$ declared a reduced excretion of sodium, whereas it resulted augmented for value $\geq 1 \%$.

Sequential Organ Failure Assessment (SOFA) score was calculated at hospital admission. The term non-critically ill denoted non-ICU admitted patients. Institutional criteria for ICU admission and/or invasive ventilation: were (a) hemodynamic instability or major electrocardiographic abnormalities; (b) sepsis with neurologic involvement or lactic 
acidosis; (c) bradypnea or apnea with respiratory arrest; (d) persistence of respiratory distress; (e) the need to protect airways due to neurological deterioration or massive secretions.

\section{Statistical analysis}

Baseline characteristics were analyzed using descriptive statistics and reported as proportions and mean (standard deviation [SD]) when appropriate. Categorical variables were analyzed using $\chi 2$ test or Fisher's test as appropriate. Analyses of continuous variables were compared using an unpaired $t$ test and Mann-Whitney test, as appropriate. Risk factors for hypokalemia were evaluated using logistic regression analysis.

Odds ratios(OR) adjusted for sex, age and SOFA score at ward admission evaluated the strength of association between hypokalemia and the risk of ICU transfer, inhospital mortality and composite outcome of ICU transfer or in-hospital mortality. A $P$ value of less than 0.05 was considered statistically significant. SPSS $23 \circledR$ was used for statistical analysis.

\section{Results}

\section{Patients}

Serum potassium level was measured in 290 (1671 samples) non-ICU admitted patients aged $64.8 \pm 13.8$ years with confirmed COVID-19. The inclusion criteria identified 171 patients with normokalemia, who were selected as a control group. Hypokalemia $(K<3.5 \mathrm{mEq} / \mathrm{L})$ was detected in 119 patients (41\%) (Fig. 1). In these subjects, serum potassium ranged from $2.4 \mathrm{mEq} / \mathrm{l}$ to $3.5 \mathrm{mEq} / \mathrm{l}$ with a mean average of $3.1( \pm 0.18) \mathrm{mEq} / \mathrm{l}$. The mean time elapsed between admission and hypokalemia was on average $3.6( \pm 5.4)$ days. One-third of cases (33.6\%) of hypokalemia were detected at admission and about half within 1 day after admission (Supplementary Fig. 1).

Demographics and clinical manifestations of patients are reported in Table 1. Most patients (90.7\%) had mild hypokalemia (serum potassium ranging from 3 to $3.4 \mathrm{mEq} / \mathrm{l}$ ). The duration of this electrolyte disturbance ranged from 1 to 13 days. Low serum potassium levels lasted on average 2.27 days per patient. Time spent on hypokalemia per number of patients (\%) was: 1 day (52\%), 2 days (11.7\%), 3 days $(18.4 \%), 4$ days (10.9\%), 5 days $(3.3 \%)$ and $>6$ days $(3.4 \%)$.

Half of the hypokalemic patients had hypocalcemia compared to $33.6 \%$ of normokalemic patients $(P=0.13)$. Serum calcium and magnesium concentration were significantly lower in hypokalemic than normokalemic patients (Table 2). Patients with hypokalemia had a longer follow-up
$(P=0.002)$ compared to normokalemic patients likely for a severe course of COVID-19, as showed by a statistically significant higher SOFA score $(P=0.014)$ at the admission. Mean serum creatinine of hypokalemic patients was $0.9 \pm 0.9 \mathrm{mg} / \mathrm{dl}$ (Table 2); the rate of acute kidney injury was similar in the two groups $(P=0.764)$.

Therapeutic agents are known to induce hypokalemia (diuretics, corticosteroids, $\beta 2$-agonists, NSAID, insulin and penicillin) were investigated in both groups (Table 1). There were no significant differences between groups in terms of prevalence.

\section{Potassium excretion}

Urine potassium-to-creatinine ratio was available only in a minority of hypokalemic patients $(n=45 ; 36.1 \%)$. A high urinary excretion of potassium (urine potassium-to-creatinine ratio $>1.5 \mathrm{mEq} / \mathrm{mmol}$ ) was documented in 43 (95.5\%) patients. Twenty-three patients $(53.4 \%)$ with high urinary potassium excretion were on diuretic therapy (furosemide) and $10(23.3 \%)$ were on corticosteroids at the time of serum $K$ measurement. The remaining 10 patients $(23.3 \%)$ with augmented kaliuresis had normal serum magnesium levels and $90 \%$ had low sodium excretion $(\mathrm{FENa}<1 \%)$ (Fig. 1). Acid-base status of these ten patients was consistent with metabolic alkalosis (70\%), respiratory alkalosis (10\%), respiratory acidosis(10\%) and normal acid-base status (10\%).

\section{Therapy in hypokalemic patients}

Potassium supplements were prescribed to correct hypokalemia (Table 2). Salts of potassium (potassium chloride) were administered via the oral route in $28(23.5 \%)$ patients and IV in $14(11.7 \%)$ patients, both oral route and IV were used in one $(0.8 \%)$ patient.

Intravenous magnesium sulfate ( $83 \mathrm{gr}$ of magnesium sulfate or $673.9 \mathrm{mEq} / \mathrm{l}$ of magnesium) was administered in 22 (18.4\%) patients for hypomagnesemia.

Regarding the total amount of K supplements, $3600 \mathrm{mq}$ (47.6 g) of potassium chloride were administered intravenously and $1536 \mathrm{mEq} / \mathrm{L}$ (20.3 gr) were administered orally. During the whole hospital stay, each potassium chloridetreated patient received a mean of 128.5 and $109.7 \mathrm{mEq}$ of potassium chloride intravenously and orally, respectively.

Potassium-sparing diuretics were largely administered among hypokalemic patients (26\%) compared to normokalemia (7\%) likely for its pharmacological action targeted to maintain a normal kalemia and prevent hypokalemia (Table 2). Forty-five (37.5\%) patients were on diuretic therapy with furosemide and two $(1.6 \%)$ with thiazide at the time of diagnosis with hypokalemia. Hypertension (57.4\%), cardiovascular disease (38.2\%) and renal impairment (31.9\%) were the potential causes of diuretic use in these patients. 
Fig. 1 Flow chart of patients' selection

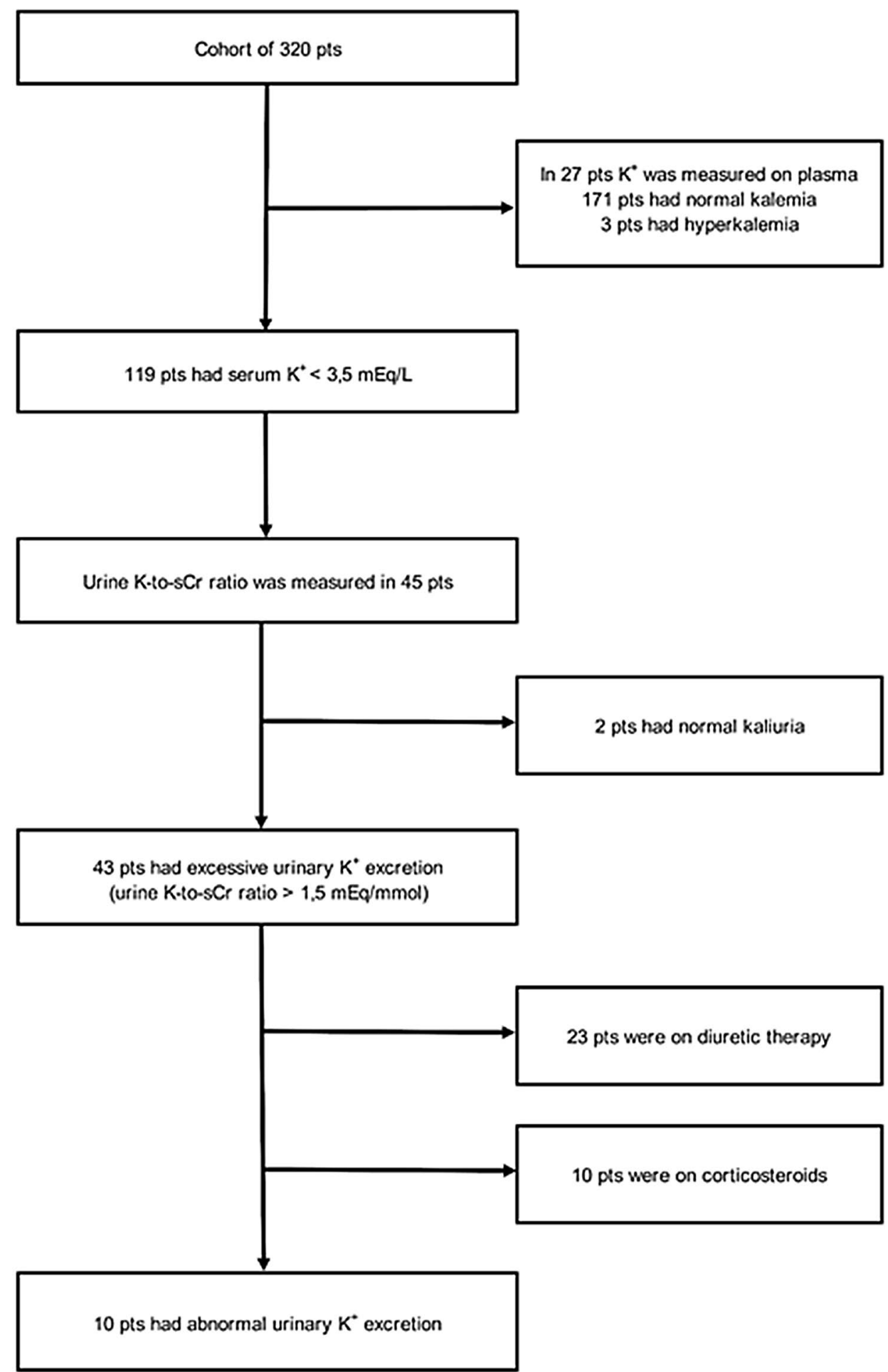

\section{Risk factors and outcome}

A chi-square test did not reveal significant differences between hypokalemic and normokaliemic patients with
$\mathrm{CPK}>1000 \mathrm{UI} / \mathrm{L}(P=0.396), \mathrm{CKP}>5000 \mathrm{UI} / \mathrm{L}(P=0.69)$ and arrhythmia $(P=0.79)$. Univariate analysis showed that female sex, corticosteroids and diuretics were significantly associated with hypokalemia in our cohort of patients 
Table 1 Demographic and clinical characteristics of hypokalemic $(K<3.5 ; \mathrm{mEq} / \mathrm{L})$ and normokaliemic $(K 3.5-5.3 \mathrm{mEq} / \mathrm{L})$ COVID-19 non-ICU admitted patients

\begin{tabular}{|c|c|c|c|}
\hline Characteristic & $\begin{array}{l}\text { Hypokalemic } \\
N=119\end{array}$ & $\begin{array}{l}\text { Normokaliemic } \\
N=171\end{array}$ & $P$ value \\
\hline Age, years $( \pm S D)$ & $65.8(12.9)$ & $64.1(14.4)$ & 0.258 \\
\hline \multicolumn{4}{|l|}{ Gender, $n(\%)$} \\
\hline Male & $73(61.3)$ & $138(80.7)$ & 0.001 \\
\hline \multicolumn{4}{|l|}{ Comorbidities, $n(\%)^{*}$} \\
\hline Diabetes & $26(27.9)$ & $24(16.3)$ & 0.113 \\
\hline Hypertension & $54(83)$ & $74(76.2)$ & 0.81 \\
\hline Cardiovascular Disease & $33(38)$ & $32(23)$ & 0.085 \\
\hline Chronic Kidney Disease & $21(21.4)$ & $29(20.4)$ & 0.875 \\
\hline \multicolumn{4}{|l|}{ Sign and symptoms, $n(\%)^{*}$} \\
\hline Dyspnea & $61(56)$ & $64(50)$ & 0.022 \\
\hline Diarrhea & $17(15.7)$ & $15(11.7)$ & 0.182 \\
\hline Cought & $66(61.1)$ & $68(53.1)$ & 0.009 \\
\hline Fatigue & $25(23.1)$ & $39(30.5)$ & 0.774 \\
\hline Myalgia & $12(11.1)$ & $8(6.3)$ & 0.098 \\
\hline Headache & $9(8.3)$ & $15(11.7)$ & 0.83 \\
\hline $\begin{array}{l}\text { From symptoms to admis- } \\
\text { sion, days }( \pm S D)\end{array}$ & $7.6(6.1)$ & $7(3.9)$ & 0.406 \\
\hline \multicolumn{4}{|l|}{ Clinical parameter, $( \pm \mathrm{SD})$} \\
\hline Systolic pressure, $\mathrm{mmHg}$ & $125(18.4)$ & $122.1(20)$ & 0.245 \\
\hline Diastolic pressure, $\mathrm{mmHg}$ & $73.3(11.7)$ & $72.8(11.3)$ & 0.911 \\
\hline Respiratory rate, n (\%) & $24.5(8.3)$ & $23(6.3)$ & 0.428 \\
\hline Baseline $\mathrm{PaO}_{2} / \mathrm{FiO}_{2}$ & $217.9(101.5)$ & $235.6(108.8)$ & 0.215 \\
\hline SOFA Score & $2.7(2.1)$ & $2.2(2.1)$ & 0.014 \\
\hline Body Temperature ${ }^{\circ} \mathrm{C}$ & $37.1(1)$ & $36.9(1)$ & 0.287 \\
\hline \multicolumn{4}{|l|}{ Acid-base status } \\
\hline $\mathrm{pH}( \pm \mathrm{SD})$ & 7.457 & 7.450 & 0.277 \\
\hline $\mathrm{CO}_{2}( \pm \mathrm{SD})$ & 40.2 & 40.4 & 0.908 \\
\hline $\mathrm{HCO}_{3}( \pm \mathrm{SD})$ & 27.2 & 26.7 & 0.360 \\
\hline Normal. $n(\%)$ & $44(40.3)$ & $68(45.6)$ & 0.713 \\
\hline Metabolic alkalosis. $n(\%)$ & $49(44.9)$ & $55(36.9)$ & 0.135 \\
\hline $\begin{array}{l}\text { Respiratory alkalosis. } \\
n(\%)\end{array}$ & $12(11)$ & $8(5.3)$ & 0.098 \\
\hline $\begin{array}{l}\text { Respiratory acidosis. } n \\
\quad(\%)\end{array}$ & $4(3.6)$ & $9(6)$ & 0.569 \\
\hline \multicolumn{4}{|l|}{ Treatment, $n(\%)^{*}$} \\
\hline Oral $\mathrm{KCl}$ supplement & $28(23,5)$ & $7(4)$ & 0.0001 \\
\hline IV $\mathrm{KCl}$ supplement & $14(11.7)$ & $3(1.7)$ & 0.0005 \\
\hline \multicolumn{4}{|c|}{ Treatment able to induce $K$ variations, $n(\%)^{*}$} \\
\hline $\begin{array}{l}\text { Potassium-sparing diuret- } \\
\text { ics }\end{array}$ & $31(26)$ & $12(7)$ & 0.0001 \\
\hline IV Mg supplement & $22(18.4)$ & $4(2.3)$ & 0.0001 \\
\hline Furosemide & $45(37.8)$ & $46(26.3)$ & 0.054 \\
\hline Tiazide & $2(1.6)$ & $2(1.1)$ & 0.713 \\
\hline Corticosteroid & $46(38.6)$ & $53(30.9)$ & 0.219 \\
\hline Insulin & $19(16.9)$ & $19(11.1)$ & 0.288 \\
\hline$\beta 2$-agonists & $6(5.04)$ & 7 (4.09) & 0.776 \\
\hline Penicillin & $17(14.2)$ & $15(8.7)$ & 0.181 \\
\hline NSAID & $5(4.2)$ & $8(4.6)$ & 1 \\
\hline
\end{tabular}

Table 1 (continued)

\begin{tabular}{llll}
\hline Characteristic & $\begin{array}{l}\text { Hypokalemic } \\
N=119\end{array}$ & $\begin{array}{l}\text { Normokaliemic } \\
N=171\end{array}$ & $P$ value \\
\hline Digoxin & 0 & $2(1.1)$ & 0.514 \\
Insulin & $19(16.9)$ & $19(11.1)$ & 0.288 \\
$\quad$ RAS-blocker & $17(14.2)$ & $20(11.6)$ & 0.592 \\
Status at the end of F-U & & & \\
Follow-up, days $( \pm \mathrm{SD})$ & $14.1(7.5)$ & $11.4(7.1)$ & $\mathbf{0 . 0 0 2}$ \\
$\quad$ Rhabdomyolysis, $\mathrm{n}(\%)$ & $2(1.7)$ & $4(2.4)$ & 1 \\
Arrhytmia, $n(\%)$ & $1(0.8)$ & $1(5.8)$ & 1 \\
$\quad$ Acute kidney disease, & $22(18.4)$ & $35(20.4)$ & 0.764 \\
$\quad n(\%)$ & $11(9.2)$ & $29(16.9)$ & 0.083 \\
ICU transfer, $n(\%)$ & $17(14.2)$ & $28(16.3)$ & 0.742 \\
$\quad$ Death of, $n(\%)$ & &
\end{tabular}

$I C U$ denotes intensive care unit, $n$ number of patients, $S D$ standard deviation, SOFA sequential organ failure assessment

* $T$-test or Mann Whitney test as appropriate; $\chi 2$ test for categorical variable

Results are reported as mean \pm SD unless otherwise stated

(Table 3). Multivariate regression model analysis showed that female sex $(P=0.003$; OR 2.44; 95\% CI 1.36-4.37) and diuretic therapy $(P=0.027$; OR $1.94 ; 95 \%$ CI $1.08-3.48)$ were significant risk factors for hypokalemia (Table 4). Survival regression analysis showed that hypokalemia adjusted for sex, age and SOFA score was not associated with transition to ICU (OR 0.52; 95\% CI 0.228-1.212, $P=0.131$ ), in-hospital mortality (OR 0.47 ; $95 \%$ CI $0.170-1.324$, $P=0.154)$ and composite outcome of ICU transfer or in-hospital mortality (OR 0.48 ; 95\% CI 0.222-1.047; $P=0,065$ ).

\section{Discussion}

Hypokalemia has been mentioned as a potential manifestation of COVID-19 [7] due to the interaction of SARS$\mathrm{CoV}-2$ with the renin-angiotensin-aldosterone system [17]. Prevalence and underlying etiologic mechanisms of hypokalemia in COVID-19 have been poorly documented. The results of our study showed that hypokalemia was a common electrolytic abnormality among COVID-19 patients. Hypokalemia was found in $41 \%$ of non-ICU admitted patients during hospitalization for severe symptoms of SARS-CoV-2 infection. It occurred in about half of the patients within the first 24 from admission and was principal of mild severity and rapid resolution. Nevertheless, administration of large amounts of potassium and magnesium supplements as well as potassium-sparing diuretics were utilized to correct low serum potassium concentrations.

We noticed that patients who experienced hypokalemia had significantly longer hospitalization than controls. Likely 
Table 2 Main lab tests of hypokalemic and normokaliemic patients at admission

\begin{tabular}{lllr}
\hline Lab test, Mean $( \pm \mathrm{SD})$ & Hypokalemic & Normokalemic & $P$ value* \\
\hline Hemoglobin, g/L & $12.1(1.6)$ & $12.8(1.793)$ & $\mathbf{0 . 0 0 1}$ \\
White cells, mm3 & $7393(5044)$ & $8156.6(8228.1)$ & 0.750 \\
Platelets, $10^{9} / \mathrm{L}$ & $252.3(119.3)$ & $243.2(116.1)$ & 0.482 \\
Glycemia, mg/dL & $105.2(35.9)$ & $110.6(54.4)$ & 0.656 \\
Potassium, mmoL/L & $3.1(0.1)$ & $4.0(0.3)$ & $<\mathbf{0 . 0 0 0 1}$ \\
Sodium, mmoL/L & $137.4(3.9)$ & $137.2(3.6)$ & 0.818 \\
Calcium, mg/L & $8.3(0.5)$ & $8.7(0.6)$ & $\mathbf{0 . 0 0 1}$ \\
Magnesium, mmoL/L & $2.1(0.3)$ & $2.6(0.3)$ & $\mathbf{0 . 0 2 8}$ \\
Chloride, mmoL/L & $99.6(4.5)$ & $100.2(4.4)$ & 0.509 \\
Creatinine, mg/ dl & $0.9(0.9)$ & $1.1(1.3)$ & $<\mathbf{0 . 0 0 0 1}$ \\
Urea, mg/dl & $55.31(47.8)$ & $52(30.3)$ & 0.564 \\
Albumin, gr/dl & $3.2(0.5)$ & $3.1(05)$ & 0.519 \\
Urine K ${ }^{+}$(spot) & $30.1(16.7)$ & $36.5(21.8)$ & 0.229 \\
Urine Creatinine (spot, mg/dL) & $66.5(79.1)$ & $84(62.7)$ & 0.477 \\
Urine protein-to-creatinine, mg/mg & $0.5(0.5)$ & $0.4(0.31)$ & 0.300 \\
D-dimer, mg/L & $2478(4070.8)$ & $3045.7(5718.3)$ & 0.826 \\
Alanine amino-transferase, U/L & $45.9(37.2)$ & $109.65(761.7)$ & 0.371 \\
Lactate dehydrogenase, U/L & $687.2(310)$ & $812.2(1914.1)$ & 0.348 \\
CPK, U/L & $556.5(1556.2)$ & $495.6(1462,1)$ & 0.739 \\
Ferritin, mg/dl & $867(416.7)$ & $856.4(824.1)$ & 0.373 \\
Bilirubin, mg/dl & $0.7(0.5)$ & $0.7(0.7)$ & 0.301 \\
C-reactive protein, mg/dl & $10.2(9.9)$ & $9.8(7.6)$ & 0.524 \\
\hline
\end{tabular}

Results are reported as mean \pm SD unless otherwise stated

* $T$-test or Mann Whitney test as appropriate. In hypokaliemic patients lab tests were obtained at diagnosis of hypokalemia
Table 3 Predictive factors of hypokalemia revealed by univariate logistic regression analysis

\begin{tabular}{lllll}
\hline & HR & CI $(95 \%)$ & & $p$ value \\
\hline Age & 1.01 & 0.99 & 1.03 & 0.31 \\
$\mathrm{PO}_{2} / \mathrm{FiO}_{2}$ & 1.00 & 0.97 & 1.00 & 0.30 \\
$\mathrm{Sex}$ & & & & \\
$\quad$ Female & 2.64 & 1.55 & 4.48 & $<\mathbf{0 . 0 0 1}$ \\
$\quad$ SOFA & 1.00 & 1.00 & 1.00 & 0.23 \\
Serum magnesium & 0.31 & 0.09 & 1.01 & 0.05 \\
Alkalosis & 1.55 & 0.94 & 2.57 & 0.09 \\
Corticosteroid & & & & \\
$\quad$ Yes/No & 1.66 & 1.01 & 2.74 & 0.05 \\
$\begin{array}{l}\text { Diuretic Therapy } \\
\quad \text { Yes/No }\end{array}$ & & & & \\
$\quad$
\end{tabular}

the significantly higher rate of respiratory symptoms (dyspnea and cough) and the worse SOFA score (2.7 vs 2.2$)$ at admission reflected a more severe systemic inflammatory response leading to a prolonged hospital stay than normokalemic patients. Multivariate analysis of risk factors for hypokalemia showed that diuretic therapy and female
Table 4 Multivariate regression model of predictive factors of hypokalemia

\begin{tabular}{|c|c|c|c|c|}
\hline \multirow[b]{2}{*}{ Alkalosis } & \multirow{2}{*}{$\begin{array}{l}\text { HR } \\
1.50\end{array}$} & \multicolumn{2}{|c|}{ CI (95\%) } & \multirow{2}{*}{$\begin{array}{l}p \text { value } \\
\mathbf{0 . 1 2 8}\end{array}$} \\
\hline & & 0.89 & 2.53 & \\
\hline Diuretic Therapy & 1.94 & 1.08 & 3.48 & 0.027 \\
\hline \multicolumn{5}{|l|}{ Sex } \\
\hline Female & 2.44 & 1.36 & 4.37 & 0.003 \\
\hline Corticosteroid & 1.25 & 0.69 & 2.27 & 0.467 \\
\hline
\end{tabular}

sex were significantly associated with a serum potassium level $<3.5 \mathrm{mEq} / \mathrm{L}$.

Diuretics are pharmacological agents largely used to treat hypertension and prevent fluid overload. Long-term administration of this class of drugs is widely known to cause hypokalemia and other electrolyte imbalances [18], as they act by inhibiting reabsorption of electrolytes in the renal tubules. A slightly higher prevalence of hypertension, cardiovascular disease and renal impairment in hypokalemic than normokalemic patients may be a plausible explanation of the higher use of diuretics in this group of patients. In addition, diuretics together with alkalosis and hypoalbuminemia 
might also be involved in causing hypocalcemia in patients with hypokalemia.

The risk of hypokalemia was up to 2.44 -fold higher in women than men. Although there are no apparent causes that interlink female sex with hypokalemia, experimental studies conducted in the 50s [19] and then confirmed in the 90s, [11] showed that women, especially aged ones, have less exchangeable body potassium than other subsets of the population. The women are, therefore, at high risk to develop hypokalemia because they have depleted deposits of potassium due to their different body composition, characterized by less amount of extracellular water compared to men.

Hypokalemia is one of the most frequent electrolyte disorders in hospitalized patients. Severe hypokalemia is associated with potentially life-threatening complications such as cardiac dysrhythmias, paralysis and rhabdomyolysis especially in subjects with underlying cardiovascular disease $[20,21]$.

We noticed a similar rate of CKP elevation ( $>1000 \mathrm{UI} / \mathrm{L})$, rhabdomyolysis $(\mathrm{CPK}>5000 \mathrm{UI} / \mathrm{L})$ or arrhythmia between hypokalemic and normokalemic patients. Hypokalemia per se was not associated with poor outcome in term of ICU transfer (OR 0.525; 95\% CI 0.228-1.212; $P=0.131$ ), in-hospital mortality (OR $0.47 ; 95 \%$ CI $0.170-1.324 ; P=0.154$ ) and composite outcome ICU transfer/in-hospital mortality (OR 0.48; 95\% CI 0.222-1.047; $P=0.065$ ). Likely, the mild severity of the disorder $(90.7 \%$ had serum levels of potassium between 3 and $3.4 \mathrm{mEq} / \mathrm{L}$ ) and the rapid recovery of normal serum potassium levels may explain the null effect of hypokalemia on major outcomes in our cohort of patients. Despite these reassuring data, serum potassium should be carefully monitored in this vulnerable subset of the population for the risk of potentially lethal arrhythmia. Hypokalemia may act by prolonging QT interval in COVID-19 patients with abnormal QT interval due to the large use of off-label agents for SARS-CoV-2 infection such as azithromycin and hydroxychloroquine. [22].

Multiple mechanisms underlying hypokalemia include diuretic therapy, gastrointestinal loss, anorexia or alkalosis [23]. More rarely, congenital or acquired tubular defect, side effects of drugs or transient tubular ischemia induce tubular potassium losses due to the disruption of renal electrolytes handling [24, 25]. Hyperaldosteronism, primitive or as a consequence of activation of the renin-angiotensin system, is also known to stimulate urinary potassium excretion. Compared to the general population, COVID-19 patients experienced at least three predisposing risk factors for hypokalemia. First, respiratory alkalosis due to hypoxiadriven hyperventilation may provoke transcellular shifts with increased intracellular uptake. Second, anorexia, as a consequence of continuative use of face mask or ventilation helmet or status of severe illness, may lead to a decrease in potassium intake. Third, diarrhea due to medication (e.g., lopinavir/ritonavir) or the cytopathic effect of the virus on the gastrointestinal cells may be a frequent cause of potassium losses from the digestive tract.

Few reports have documented an interplay between hypokalemia and coronavirus infection so far. Hypokalemia has been reported during the SARS-CoV-1 outbreak in Canada. Here, Booth et al. [9] reported a low potassium level in 62 out of 144 (43\%) patients (mean serum potassium, $3.2 \mathrm{mEq} / \mathrm{l}$ ) during hospitalization. Hypokalemia was not isolated and occurred with other electrolyte abnormalities such as hypomagnesemia and hypophosphatemia. The authors were unable to establish clear evidence for electrolyte imbalance and suggested that it may be a clinical manifestation of the infection or a nephrotoxic effect of ribavirin or other medicaments on renal electrolytes handling. The abstract of a Chinese article, reported hypokalemia as a side effect of glucocorticoids in SARS-CoV-1 infected patients [10]. These agents increase urinary potassium excretion through activation of the mineralocorticoid receptor on the renal tubular cells [26]. A recent study conducted on 179 COVID-19 patients admitted to the hospital in Wenzhou, China, reported that hypokalemia was a common electrolytic disorder and affected 55\% of patients. Hypokalemic patients had an increased urinary $K$ output compared with the normal group with normokalemia. Based on these results, the authors suggested that the primary cause of hypokalemia was consistent with the disruption of ACE2 by the binding of SARS-CoV-2. [27]

To evaluate tubular potassium handling in our cohort of COVID-19 patients, we investigated the magnitude of potassium secretion in a group of hypokalemic patients with available urine electrolytes. In line with the findings of the above-mentioned study [27], urine potassium-to-creatinine ratio was increased in $95.5 \%$ of patients who underwent urinary examination $(n=45)$. In about three-fourths of the urinary sample, the high urinary $K$ excretion was associated with diuretic and corticosteroid therapy (kaliuretic agents), whereas causes were unclear in the remaining patients $(n=10,23.3 \%)$.

Bringing it all together, etiology of hypokalemia in our COVID-19 patients seems multifactorial and is likely associated with augmented urinary loss. Cytopathic effect of SARS-CoV-2 infection [11] and the indirect effect of cytokines triggered by viral infection [28] on renal tubules may induce a COVID-19-related potassium-losing tubulopathy. However, determining the exact etiology of hypokalemia is challenging in the presence of multiple interfering effects from therapeutic agents. Selection bias arising from the retrospective nature of our study, absence of a control group in the evaluation of urinary potassium excretion and lack of plasma renin and aldosterone measurements evaluating the activity of the RASS further limited risk assessment for hypokalemia in our study. Despite 
these limitations, we collected comprehensive data on all prescribed medications and accompanying acid-base disorders that provided relevant information on key determinants of hypokalemia in COVID-19.

\section{Conclusions}

Hypokalemia was a frequent electrolyte disorder in hospitalized patients with COVID-19. It was principally of mild severity and of rapid solution. Our findings support the need to frequently measure the level of serum potassium along with urine potassium-to-creatinine ratio or $24 \mathrm{~h}$ potassium excretion in COVID-19 patients to improve the complex management of these subjects. Low potassium level should be supplemented per os or IV, and a careful assessment of the ECG should be performed, especially in concomitance of potentially arrhythmogenic drugs.

Although hypokalemia was not associated with mortality in our cohort of patients, it may be a life-threatening condition (e.g., in long QT patients) if it remains untreated.

Acknowledgements \#Modena Covid-19 Working Group (MoCo19) includes: Cristina Mussini, Giovanni Guaraldi, Erica Bacca, Andrea Bedini, Vanni Borghi, Giulia Burastero, Federica Carli, Giacomo Ciusa, Luca Corradi, Gianluca Cuomo, Margherita Di Gaetano, Giovanni Dolci, Matteo Faltoni, Riccardo Fantini, Giacomo Franceschi, Erica Franceschini, Vittorio Iadisernia, Damiano Larné, Marianna Menozzi, Marianna Meschiari, Jovana Milic, Gabriella Orlando, Francesco Pellegrino, Alessandro Raimondi, Carlotta Rogati, Antonella Santoro, Roberto Tonelli, Marco Tutone, Sara Volpi, and Dina Yaacoub (Infectious Diseases Clinics, University Hospital, via del Pozzo 71, 41124 Modena, Italy); Gianni Cappelli, Riccardo Magistroni, Gaetano Alfano, Francesco Fontana, Ballestri Marco, Giacomo Mori, Roberto Pulizzi, Elisabetta Ascione, Marco Leonelli, Francesca Facchini, Francesca Damiano, (Nephrology Dialysis and Transplant Unit, University Hospital of Modena, Modena, Italy); Massimo Girardis, Alberto Andreotti, Emanuela Biagioni, Filippo Bondi, Stefano Busani, Giovanni Chierego, Marzia Scotti, and Lucia Serio (Department of Anesthesia and Intensive Care, University Hospital, via del Pozzo 71, 41124 Modena, Italy); Andrea Cossarizza, Caterina Bellinazzi, Rebecca Borella, Sara De Biasi, Anna De Gaetano, Lucia Fidanza, Lara Gibellini, Anna Iannone, Domenico Lo Tartaro, Marco Mattioli, Milena Nasi, Annamaria Paolini, and Marcello Pinti (Chair of Pathology and Immunology, University of Modena and Reggio Emilia, Via Campi, 287, 41125 Modena, Italy).

Funding This study was not funded.

\section{Compliance with ethical standards}

Conflicts of interest The authors have no conflicts of interest to declare.

Ethical approval This manuscript is a preprint article published with the following https://doi.org/10.1101/2020.06.14.20131169 [29]. The study has been conducted ethically in accordance with the World
Medical Association Declaration of Helsinki. The Ethic Committee of Region Emilia Romagna approved the study.

\section{References}

1. COVID-19 Map;Coronavirus COVID-19 Global Cases by the Center for Systems Science and Engineering (CSSE) at Johns Hopkins University (JHU) [Internet]. Johns Hopkins Coronavirus Resour Cent. [cited 2020 May 15]. https://coronavirus.jhu.edu/ map.html

2. Wu Z, McGoogan JM. Characteristics of and important lessons from the coronavirus disease 2019 (COVID-19) outbreak in China: summary of a report of 72314 cases from the chinese center for disease control and prevention. JAMA. 2020;323(13):1239-42.

3. Guan W, Ni Z, Hu Y, Liang W, Ou C, He J, et al. Clinical Characteristics of Coronavirus Disease 2019 in China. N Engl J Med. $2020 \mathrm{Feb} ; 0(0)$ :null.

4. Wang T, Du Z, Zhu F, Cao Z, An Y, Gao Y, et al. Comorbidities and multi-organ injuries in the treatment of COVID-19. The Lancet. 2020;395(10228):e52.

5. Moore BJB, June $\mathrm{CH}$. Cytokine release syndrome in severe COVID-19. Science. 2020. https://doi.org/10.1126/science.abb89 25.

6. Garg S. Hospitalization Rates and Characteristics of Patients Hospitalized with Laboratory-Confirmed Coronavirus Disease 2019COVID-NET, 14 States, March 1-30, 2020. MMWR Morb Mortal Wkly Rep. 2020;69. DOI: https://doi.org/10.15585/mmwr.mm691 $5 \mathrm{e} 3$

7. Bansal M. Cardiovascular disease and COVID-19. Diabetes Metab Syndr Clin Res Rev. 2020;14(3):247-50.

8. Basu-Ray I, Soos MP. Cardiac Manifestations Of Coronavirus (COVID-19). StatPearls. Treasure Island (FL): StatPearls Publishing; 2020; [cited 2020 May 30]. http://www.ncbi.nlm.nih.gov/ books/NBK556152/

9. Booth CM, Matukas LM, Tomlinson GA, Rachlis AR, Rose DB, Dwosh HA, et al. Clinical features and short-term outcomes of 144 patients with SARS in the greater Toronto area. JAMA. 2003;289(21):2801-9.

10. Li N, Wang G, Wu Y, Xie G, Xiao F, Chen B, et al. Side effects of glucocorticosteroids in the management of 1291 patients of SARS. Beijing Da Xue Xue Bao. 2004;36(5):519-24.

11. Su H, Yang M, Wan C, Yi L-X, Tang F, Zhu H-Y, et al. Renal histopathological analysis of 26 postmortem findings of patients with COVID-19 in China. Kidney Int. 2020 Apr;0(0). DOI: https ://doi.org/10.1016/j.kint.2020.04.003

12. WHO/COVID-19/laboratory/2020.5. Laboratory testing for 2019 novel coronavirus (2019-nCoV) in suspected human cases [Internet]. [cited $2020 \mathrm{Apr}$ 12]. https://www.who.int/publicatio ns-detail/laboratory-testing-for-2019-novel-coronavirus-in-suspe cted-human-cases-20200117

13. Kidney Disease: Improving Global Outcomes (KDIGO) Acute kidney injury work group. KDIGO Clinical practice guideline for acute kidney injury. Kidney inter., Suppl. 2012; 2: 1-138. $2012 ; 141$.

14. Kardalas E, Paschou SA, Anagnostis P, Muscogiuri G, Siasos G, Vryonidou A. Hypokalemia: a clinical update. Endocr Connect. 2018;7(4):R135-46.

15. Yalamanchili HB, Calp-Inal S, Zhou XJ, Choudhury D. Hypokalemic nephropathy. Kidney Int Rep. 2018;3(6):1482-8.

16. Jędrusik P, Symonides B, Wojciechowska E, Gryglas A, Gaciong Z. Diagnostic value of potassium level in a spot urine sample as an index of 24-h urinary potassium excretion in 
unselected patients hospitalized in a hypertension unit. PLoS ONE. 2017;12(6):e0180117.

17. Alfano G, Guaraldi G, Fontana F, Ferrari A, Magistroni R, Mussini $\mathrm{C}$, et al. The role of the renin-angiotensin system in severe acute respiratory syndrome-CoV-2 infection. Blood Purif. 2020 $1-5$.

18. Arampatzis S, Funk G-C, Leichtle AB, Fiedler G-M, Schwarz C, Zimmermann $\mathrm{H}$, et al. Impact of diuretic therapy-associated electrolyte disorders present on admission to the emergency department: a cross-sectional analysis. BMC Med. 2013;11:83.

19. Sagild U. Total exchangeable potassium in normal subjects with special reference to changes with age. Scand J Clin Lab Invest. 1956;8(1):44-50.

20. Bowling CB, Pitt B, Ahmed MI, Aban IB, Sanders PW, Mujib M, et al. Hypokalemia and outcomes in patients with chronic heart failure and chronic kidney disease: findings from propensitymatched studies. Circ Heart Fail. 2010;3(2):253-60.

21. Goyal A, Spertus JA, Gosch K, Venkitachalam L, Jones PG, den Berghe GV, et al. Serum potassium levels and mortality in acute myocardial infarction. JAMA. 2012;307(2):157-64.

22. Gennari FJ. Disorders of potassium homeostasis. Hypokalemia and hyperkalemia. Crit Care Clin. 2002 18(2):273-88, vi.

23. Paice BJ, Paterson KR, Onyanga-Omara F, Donnelly T, Gray JM, Lawson DH. Record linkage study of hypokalaemia in hospitalized patients. Postgrad Med J. 1986;62(725):187-91.
24. Asmar A, Mohandas R, Wingo CS. A physiologic-based approach to the treatment of a patient with hypokalemia. Am J Kidney Dis Off J Natl Kidney Found. 2012;60(3):492-7.

25. Unwin RJ, Luft FC, Shirley DG. Pathophysiology and management of hypokalemia: a clinical perspective. Nat Rev Nephrol. 2011;7(2):75-84.

26. Palmer BF. Regulation of potassium homeostasis. Clin J Am Soc Nephrol CJASN. 2015;10(6):1050-60.

27. Chen D, Li X, Song Q, Hu C, Su F, Dai J, et al. Assessment of Hypokalemia and Clinical Characteristics in Patients With Coronavirus Disease 2019 in Wenzhou, China. JAMA Netw Open. 2020;3(6). DOI: https://doi.org/10.1001/jamanetworkopen .2020 .11122

28. Holdsworth SR, Gan P-Y. Cytokines: names and numbers you should care about. Clin J Am Soc Nephrol CJASN. 2015;10(12):2243-54.

29. Alfano G, Ferrari A, Fontana F, Perrone R, Mori G, Ascione E, et al. Hypokalemia in Patients with COVID-19. medRxiv. 2020;2020.06.14.20131169.

Publisher's Note Springer Nature remains neutral with regard to jurisdictional claims in published maps and institutional affiliations.

\section{Affiliations}

\section{Gaetano Alfano ${ }^{1,2,3} \cdot$ Annachiara Ferrari $^{1} \cdot$ Francesco Fontana $^{2} \cdot$ Rossella Perrone $^{1} \cdot$ Giacomo Mori $^{2}$. Elisabetta Ascione ${ }^{2}$. Riccardo Magistroni ${ }^{1,2}$. Giulia Venturi ${ }^{4}$. Simone Pederzoli ${ }^{5}$. Gianluca Margiotta ${ }^{5}$.

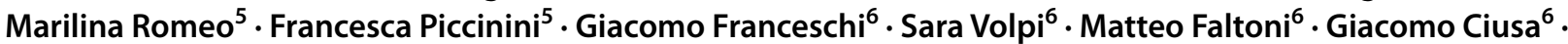 Erica Bacca ${ }^{6} \cdot$ Marco Tutone $^{6}$. Alessandro Raimondi ${ }^{6} \cdot$ Marianna Menozzi $^{6}$. Erica Franceschini ${ }^{6}$. Gianluca Cuomo ${ }^{6}$. Gabriella Orlando $^{6}$. Antonella Santoro ${ }^{6}$. Margherita Di Gaetano ${ }^{6}$. Cinzia Puzzolante ${ }^{6} \cdot$ Federica Carli $^{6}$. Andrea Bedini ${ }^{6}$. Jovana Milic ${ }^{3,6} \cdot$ Marianna Meschiari $^{6} \cdot$ Cristina Mussini $^{6} \cdot$ Gianni Cappelli ${ }^{1,2} \cdot$ Giovanni Guaraldi $^{6}$ on behalf of the Modena Covid-19 Working Group (MoCo19)}

1 Surgical, Medical and Dental Department of Morphological Sciences, Section of Nephrology, University of Modena and Reggio Emilia, via del Pozzo 71, 41124 Modena, Italy

2 Nephrology, Dialysis and Transplant Unit, University Hospital of Modena, Modena, Italy

3 Clinical and Experimental Medicine Ph.D. Program, University of Modena and Reggio Emilia, Modena, Italy
4 Department of Biomedical, Metabolic and Neural Sciences, Section of Clinical Neurosciences, University of Modena and Reggio Emilia, Modena, Italy

5 Unit of Endocrinology, Department of Biomedical, Metabolic and Neural Sciences, University of Modena and Reggio Emilia,, Modena, Italy

6 Clinic of Infectious Diseases, University Hospital of Modena, Modena, Italy 